\title{
Strain Elastography \& Tissue Characterization as a Tool to Differentiate Tumor from Healthy Tissue
}

\author{
Mahdi Al-Qahtani, Omar Altuwaijri and Ravish Javed \\ Biomedical Technology Department, College of Applied Medical Sciences, King Saud University, \\ Riyadh, KSA \\ amahdi@ksu.edu.sa; oaltwijri@ksu.edu.sa; ravish.javed@yahoo.com
}

\begin{abstract}
The purpose of this study was to compare diagnostic values of normal and effected tissues with two techniques using strain elastography and tissues characterization. This study was carried out on a breast phantom containing all human body parameters. Analysis was performed using a lone phantom to correlate a relation between the values of Strain Elastography (SE) and first order texture parameters results. For SE SonixTouch Q+ (Ultrasonix Medical Corporation, 130-4311 Viking Way, Richmond, Canada) device using a linear-array ultrasound probe at a frequency of $10 \mathrm{MHz}$ with a gain of $40 \%$. Elastography breast phantom was purchased from CAE healthcare USA, 3600 Edgelake Drive Sarasota FL, USA. For tissue characterization a Region of Interest (ROI) that encompasses both (normal and stiffer) areas were selected. MAZDA software was used to carry out the image analysis (mean and variance) of the tumour and healthy tissue, ROI of 1600 pixels at both regions was selected. An affirmative and resilient outcome was observed between the numerals of normal and tumor tissues, both for SE and first order texture parameters values. After our study we suggest that SE and tissue characterisation via first order texture parameter is a reliable technique to highlight normal and tumor tissue (with respect to same reference, for SE technique only). SE and first order texture parameters (mean and variance) paved way in highlighting the breast tumors fully. It is suggested that SE being more reliable approach in determining the stiffness for breast lesion, as it produces the results with real time imaging. However texture parameter gives an objective assessment of the image with a discriminating feature of the tissue.
\end{abstract}

Keywords: Elastography, Texture analysis, Breast, Tumor.

\section{Introduction}

Cancer in breast is one of the most common cause of death among women worldwide [1]. Breast tumor frequency has increased rapidly in recent years, it is the most common pathological formation towards breast cancer [2]. During reproductive years, its incidence increases in particular and after 50 years of age with a slower rate (average age of menopause) [3]. Occurrence rates of breast cancer are high in women across developed countries in contrast to less developed countries, but frequency of incidence rates are also increasing in less developed countries [3]. About 2.7\% of women in Europe and North 
America suffers from breast cancer by age of 55 , whereas $5.0 \%$ by age of 65 and around $7.7 \%$ by age of $75[4]$.

Variation in pliability of tissues is ascribed to pathological condition [5]. Tissue elasticity changes due to numerous circumstances, among cancerous tissues appear as hard lumps due to density increased, whereas other tissues disorders encompasses deposition due to fat or collagen. In conventional ultrasound examination cysts containing fluid may not be visible. A diminished superficial lesion or pathological abnormality departs undetected via traditional ultrasound in several cases [6]. Conventional ultrasound is based on qualitative technique [7], though to differentiate healthy tissues from the diseased there was a need to establish a quantitative approach. The ultrasound elastography was introduced to overcome this issue.

Elastography is a non-invasive medical imaging technique. It analyses stiffness of effected tissue region when compared to other normal tissue as per the image, based on this comparisons it detects tumours. Numerous imaging mode of ultrasound is been used for the most common type of elastography to equate the shape of the tissue under examination before and after it is compressed slightly. As cancerous tissue tends to be stiffer than normal tissue. Image obtained by elastogram shows different shades of light and dark, with different degree of stiffness. Several tumours are detected with elastogram, including breast tumor better than traditional ultrasonic images. Elastography use a small uniform compression on the tissue that provides insight of the elastic properties of the tissue [8].

Two basic types of elastography are currently performed; strain elastography (SE) and shear wave elastography (SWE) [9]. SE is observed by applying strain manually by the user and establishing the relative strain in a tissue with comparisons to its surrounding tissue $[10,11]$. Whereas, SWE simply uses a combination of shear waves, it measure the absolute value of the tissue and delivers tissue elasticity based on force values detected by the machine $[12,13]$.

Recent studies have demonstrated SE techniques individually for various tissues. However, till date their results are not studied and supported via texture parameters in particular. Here we report the evaluation of breast tumor with healthy tissue in a phantom using SE and institute a link by analyzing the image via first order texture parameter.

\section{Material and Methods}

\subsection{Study design}

This study was conducted in the Biomedical Technology Department in accordance with guidelines followed by ethical review board of King Saud University. A breast phantom was utilized for the study containing all human body parameters. Analysis was performed using a lone phantom to correlate a relation between the values of SE and first order texture parameters results.

\subsection{Ultrasound examination}

B-Mode ultrasound on breast phantom was performed with using SonixTouch Q+ (Ultrasonix Medical Corporation, 130-4311 Viking Way, Richmond, Canada) device using a linear-array ultrasound probe at a frequency of $10 \mathrm{MHz}$ with a gain of $40 \%$. Elastography breast phantom was purchased from CAE healthcare USA, 3600 Edgelake Drive Sarasota FL, USA. The breast phantom ultrasound examination was 
Mahdi Al-Qahtani, Omar Altuwaijri and Ravish Javed; Strain Elastography \& Tissue Characterization as a Tool to Differentiate Tumor from Healthy Tissue. Journal of Biomedical Engineering and Medical Imaging, Volume 5, No 1, February (2018), pp 17-24

performed via previously described guidelines as per The World Federation for Ultrasound in Medicine and Biology (WFUMB) [14].

\subsection{Elastographic strain ratio}

Elastography mode was selected to measure strain ratio on two different regions on the phantom, tumour and adjacent normal tissue with a common normal tissue (tissue A) as a reference within the phantom was castoff to measure strain ratios. Primarily strain ratio for tumour was calculated by taking a region on interest (ROI) positioned in the tumour with tissue $A$. Furthermore for calculating the second strain ratio another $\mathrm{ROI}$ normal tissue (adjacent to tumour) was also examined with same tissue $A$.

\subsection{Image acquisition}

A set of one best image within the range of strain sensor from a cine loop of 99 images were picked. In each image 5 random areas were selected for strain ratios, initially for tumour then adjacent healthy tissue with respect to a common tissue $A$ as shown in figure 1 . To increase reliability elastographic strain ratios were repeated in 5 successive sessions, with atleast a 2 -hours interval.

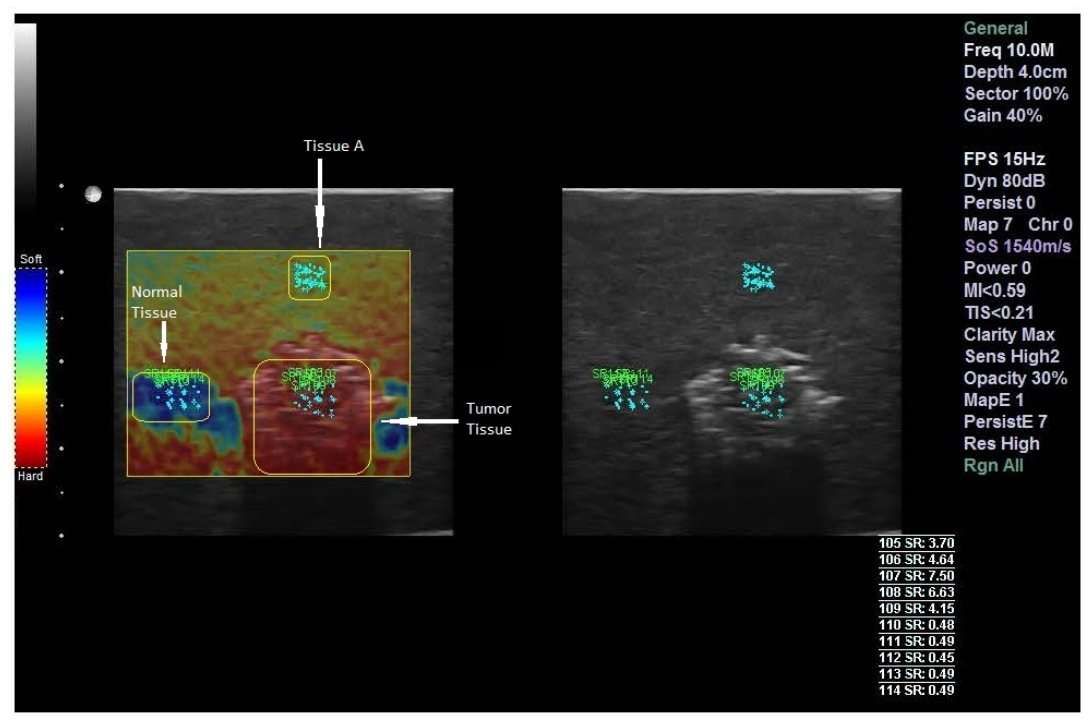

Figure 1 Elastographic and B-Mode image of breast phantom with Normal, Tumor and Tissue A (as reference).

\subsection{First order texture parameters}

To correlate the tumour and healthy tissue on the breast phantom an ROI that encompasses both the areas were selected. MAZDA version 4.5 software (Institute of Electronics, Technical University of Lodz, Poland) was used to perform the texture feature extraction, it permits calculation of the texture features suggested in the available literature. To carry out the image analysis of the tumor and healthy tissue, ROI of 1600 pixels at both regions was selected. All 5 images at both (normal and stiffer) sections were analysed for mean and variance with same ROI. The intensity of the signal of each ROI was normalized using the limitation of dynamics to $\mu \pm 3 \sigma$ ( $\mu$, gray-level mean; and $\sigma$, gray-level standard deviation). 


\subsection{Statistical analysis}

The data obtained from texture parameter were analysed with SPSS version 2 software (IBM Corporation, Armonk, NK). One way ANOVA was utilized to ascertain the significant difference between the values. Statistical significance was set at $P$ equal 0.05 .

\section{Results}

An affirmative and resilient outcome was observed between the numerals of normal and tumor tissues, both for SE and first order texture parameters values.

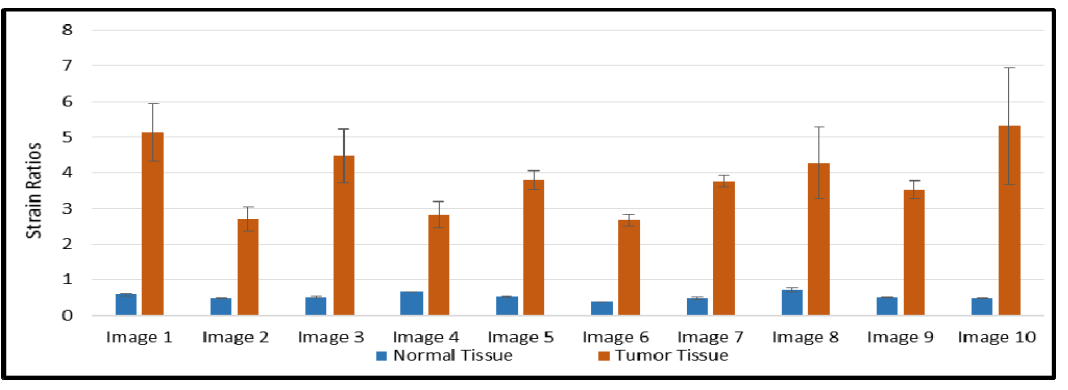

Figure 2 Strain ratios of individual images of normal tissue with respect to tumor.

Figure 2 represents strain ratios of each image using ultrasound elastography technique with persistent trend of superior stiffness in the tumor region in contrast to normal tissue. Image 10 showing the highest strain ratios, whereas Image $2 \& 6$ showing the lowest value of stiffness in tumor.



Figure 3 Mean values of each image with normal and tumor tissues.

Figure 3 depicts the mean values of each image for both the tissues (normal and tumor) via using first order texture parameters. The mean value from texture parameter illustrates irregularity in value of hardness of tumour tissue when compared to normal tissue.

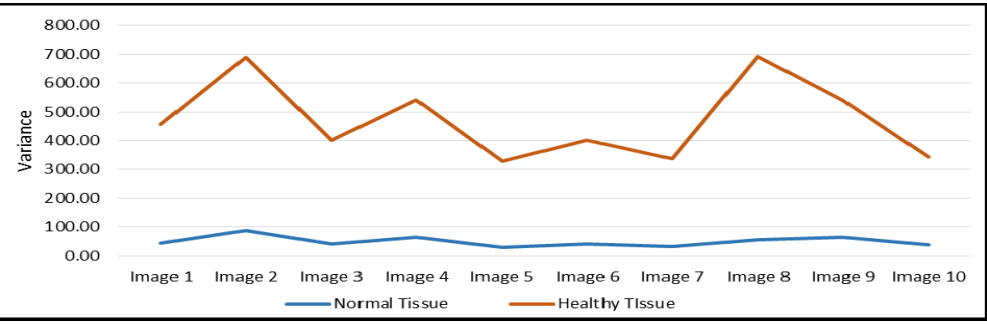

Figure 4 Variance values of individual image with normal and tumor tissues. 
Mahdi Al-Qahtani, Omar Altuwaijri and Ravish Javed; Strain Elastography \& Tissue Characterization as a Tool to Differentiate Tumor from Healthy Tissue. Journal of Biomedical Engineering and Medical Imaging, Volume 5, No 1, February (2018), pp 17-24

Figure 4 displays the values of variance for each image encompassing normal and tumor tissues through first order texture parameters. A significant difference and indiscretion in values of tumour tissues was perceived when associated with normal tissue.

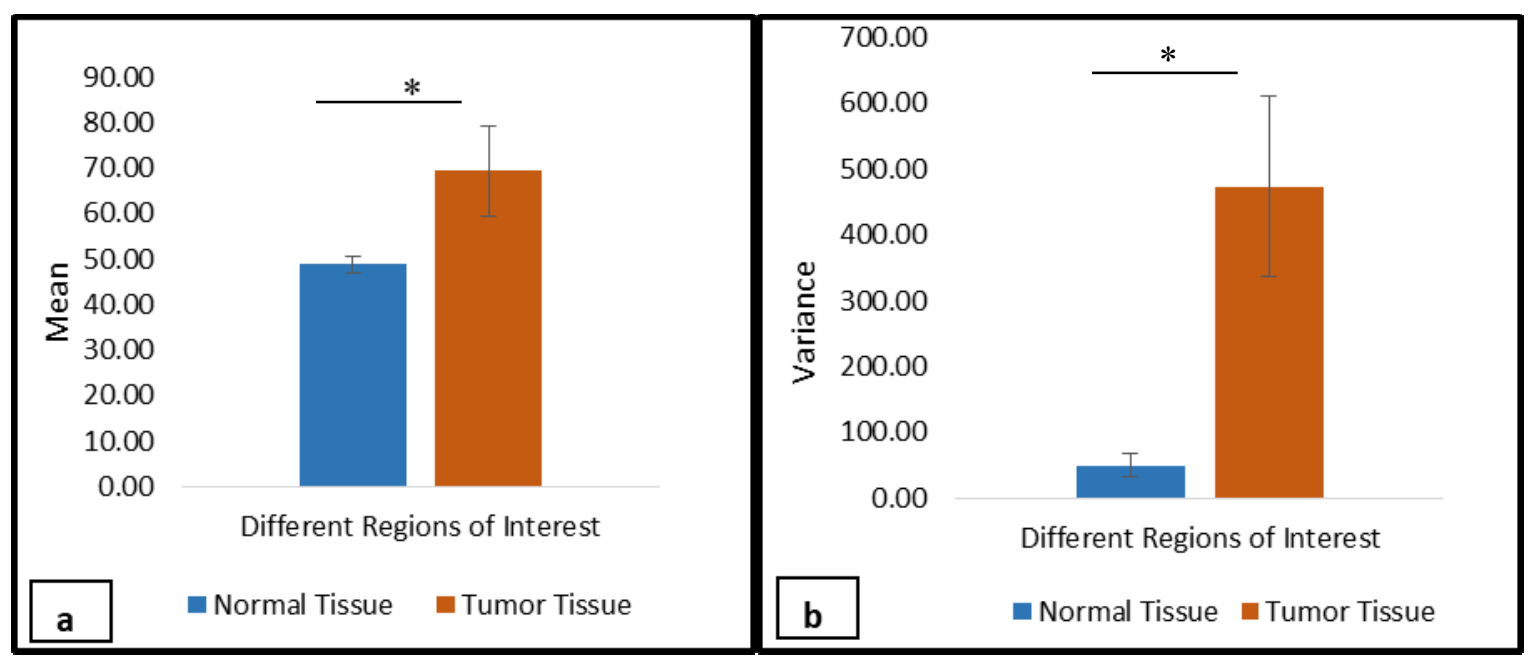

Figure 5 (a) Cumulative mean values of normal and tumor tissues. (b) Cumulative variance values of normal and tumor tissues. * Showing significant difference between values of tissues.

Figure 5 a \& b illustrates the cumulative mean and variance values of all the images consisting normal and tumor tissues, respectively. As seen evidently both the figure show a higher value of mean and variance for tumor tissue when compared to normal tissue.

\section{Discussion}

Lesion in breast lump is a common disorder paving way for tumors. Often these tumor can get malignant and if not separated from breast and can transform to cancer in breast [15]. Imaging is evident for evaluation purposes when there are obstinate or perplexing symptoms [16-18]. SE and magnetic resonance imaging are the most extensively used techniques for identifying the pathological status of the tumor. SE is a practical and non-invasive method. Furthermore, with the introduction of high frequency transducers has ensued in better visual appearance of the superficial organs and especially soft tissues [19]. The results of SE are the imaging methodology with higher reliability and accuracy. To determine deviation in hyperechoic areas for swelling, tears, lesions and calcifications in tissues with the help of SE using high-frequency transducers is quite successful. Though, conventional ultrasound imaging is yet to advance the desired accuracy rates as SE in displaying out the abnormalities. SE exhibits a comparative value of strain in contrast to the surrounding area [20]. This approach is achieved in realtime with either manually or automatically compressing the desired tissue [21,22]. Sensation practice is widely used since decades to perceive stiffness change in a tissue. SE also work on the origin of sensation [9]. For calculating the stiffness through SE two ROI's are selected to display the value. Notable studies are already been performed on breast, liver, thyroid and other tissues [23-27]. SE is user dependent and occasionally variations in result are also common [28]. 


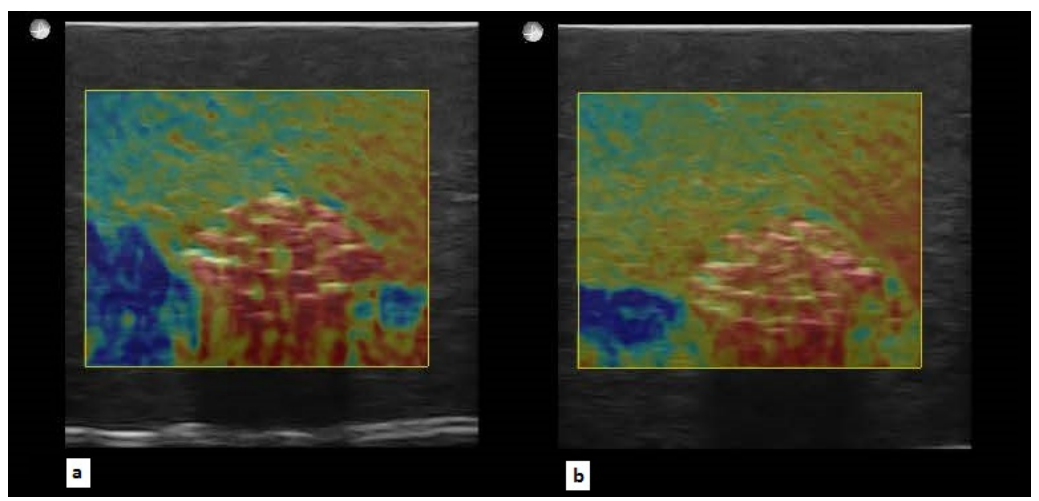

Figure 6 a \& b Two different elastographic image of same phantom with clear visual difference.

As we can see in figure 6 a \& b shows two different elastographic images of the same breast phantom. As perceived from the images in figure $6 a \& b$ there is a slight variation in values of strain ratios as well as different visual pattern of normal and tumor tissues. Despite the difference in values both the figures are clearly showing tumor as red in colour with higher stiffness while soft tissue can be seen with blue colour with lesser stiffness.

Texture parameters studies have been conducted on various instances for numerous body parts images. However texture parameter studies on breast phantom consolidating SE was falling short. Here we determine first order texture parameters by analysing the values of mean and variance show remarkable difference among normal and tumor tissue, also assisting the values of strain ratios for both the tissues (normal and tumor). A higher significant difference value of variance was detected for normal and tumor tissue, whereas a low significant difference value of mean was observed.

After our study we suggest that SE and tissue characterization via first order texture parameter is a reliable technique to highlight normal and tumor tissue (with respect to same reference, for SE technique).

\section{Conclusion}

SE and first order texture parameters (mean and variance) paved way in highlighting the breast tumors fully. It is suggested that SE being more reliable approach in determining the stiffness for breast lesion, as it produces the results with real time imaging. However texture parameter gives an objective assessment of the image with a discriminating feature of the tissue.

Current study was exercised on a breast phantom containing all human body parameters. It is recommended that future studies must be conducted on females suffering from tumor in breast with respect to surrounding normal tissue, to measure the strain ratio for stiffness with suitable external reference with values of mean and variances for first order texture parameter.

\section{ACKNOWLEDGEMENT}

The authors would like to extend their appreciation to the College of Applied Medical Sciences Research Center and the Deanship of Scientific Research at King Saud University for funding this research. 
Mahdi Al-Qahtani, Omar Altuwaijri and Ravish Javed; Strain Elastography \& Tissue Characterization as a Tool to Differentiate Tumor from Healthy Tissue. Journal of Biomedical Engineering and Medical Imaging, Volume 5, No 1, February (2018), pp 17-24

\section{REFERENCES}

[1] Murray, C.J. and A.D. Lopez, Mortality by cause for eight regions of the world: Global Burden of Disease Study. The lancet, 1997. 349(9061): p. 1269-1276.

[2] Carter, C.L., C. Allen, and D.E. Henson, Relation of tumor size, lymph node status, and survival in 24,740 breast cancer cases. Cancer, 1989. 63(1): p. 181-187.

[3] Key, T.J., P.K. Verkasalo, and E. Banks, Epidemiology of breast cancer. The lancet oncology, 2001. 2(3): p. 133-140.

[4] Cancer, C.G.o.H.F.i.B., Breast cancer and hormone replacement therapy: collaborative reanalysis of data from 51 epidemiological studies of 52705 women with breast cancer and 108411 women without breast cancer. The Lancet, 1997. 350(9084): p. 1047-1059.

[5] Krouskop, T.A., et al., Elastic moduli of breast and prostate tissues under compression. Ultrasonic imaging, 1998. 20(4): p. 260-274.

[6] Ophir, J., et al., Elastography: a quantitative method for imaging the elasticity of biological tissues. Ultrasonic imaging, 1991. 13(2): p. 111-134.

[7] Manning, F.A., C.M. Hill, and L.D. Platt, Qualitative amniotic fluid volume determination by ultrasound: antepartum detection of intrauterine growth retardation. American journal of obstetrics and gynecology, 1981. 139(3): p. 254-258.

[8] Ophir, J., et al., Elastography: ultrasonic estimation and imaging of the elastic properties of tissues. Proceedings of the Institution of Mechanical Engineers, Part H: Journal of Engineering in Medicine, 1999. 213(3): p. 203-233.

[9] Bamber, J., et al., EFSUMB guidelines and recommendations on the clinical use of ultrasound elastography. Part 1: Basic principles and technology. Ultraschall in der Medizin-European Journal of Ultrasound, 2013. 34(02): p. 169-184.

[10] Vural, M., et al., The evaluation of the retrobulbar orbital fat tissue and optic nerve with strain ratio elastography. Medical ultrasonography, 2015. 17(1): p. 45.

[11] Park, H., et al., Strain elastography features of epidermoid tumours in superficial soft tissue: differences from other benign soft-tissue tumours and malignant tumours. The British journal of radiology, 2015. 88(1050): p. 20140797.

[12] Cortes, D.H., et al., Continuous shear wave elastography: a new method to measure viscoelastic properties of tendons in vivo. Ultrasound in Medicine and Biology, 2015. 41(6): p. 1518-1529.

[13] Athanasiou, A., et al., Feasibility of imaging and treatment monitoring of breast lesions with threedimensional shear wave elastography. Ultraschall in der Medizin-European Journal of Ultrasound, 2017. 38(01): p. 51-59.

[14] Barr, R.G., et al., WFUMB guidelines and recommendations for clinical use of ultrasound elastography: Part 2: breast. Ultrasound in Medicine and Biology, 2015. 41(5): p. 1148-1160. 
[15] Rickert, R.R., L. Kalisher, and R.V. Hutter, Indurative mastopathy: a benign sclerosing lesion of breast with elastosis which may simulate carcinoma. Cancer, 1981. 47(3): p. 561-571.

[16] Gordon, P.B. and S.L. Goldenberg, Malignant breast masses detected only by ultrasound. A retrospective review. Cancer, 1995. 76(4): p. 626-630.

[17] Hayes, R., M. Michell, and H. Nunnerley, Acute inflammation of the breast-the role of breast ultrasound in diagnosis and management. Clinical Radiology, 1991. 44(4): p. 253-256.

[18] Houssami, N., et al., Sydney Breast Imaging Accuracy Study: comparative sensitivity and specificity of mammography and sonography in young women with symptoms. American Journal of Roentgenology, 2003. 180(4): p. 935-940.

[19] Rizzatto, G., et al., High-resolution sonography of breast carcinoma. European journal of radiology, 1997. 24(1): p. 11-19.

[20] Al-Qahtani, M., Shear-Wave and Strain Elastography: A Comparative Review on Principles, Basic Techniques and Applications. Current Medical Imaging Reviews, 2016. 12(4): p. 269-278.

[21] Cho, N., et al., Sonoelastographic strain index for differentiation of benign and malignant nonpalpable breast masses. Journal of Ultrasound in Medicine, 2010. 29(1): p. 1-7.

[22] Hiltawsky, K.M., et al., Freehand ultrasound elastography of breast lesions: clinical results. Ultrasound in Medicine and Biology, 2001. 27(11): p. 1461-1469.

[23] Al-Qahtani, M., et al., A Comparative Study of Shear-Wave Elastography and Strain Elastography on a Breast Phantom for Diagnosis of Tumor and Cyst. Journal of Biomedical Engineering and Medical Imaging, 2015. 2(3): p. 24.

[24] Monpeyssen, H., et al., Elastography of the thyroid. Diagnostic and interventional imaging, 2013. 94(5): p. 535-544.

[25] Rykhtik, P., et al., Experience of Using Elastography in the Diagnosis of Liver Fibrosis in the Practice of Hepatology Center. Radiology, 2015. 1: p. 49.

[26] Nicolas, E., S. Callé, and J.-P. Remenieras, Generating shear waves in the human brain for ultrasound elastography: a new approach. Physics Procedia, 2015. 70: p. 1255-1259.

[27] Uramoto, H., et al., Intraoperative ultrasound elastography has little diagnostic benefit for deeper tumours of the lung. European Journal of Cardio-Thoracic Surgery, 2015. 49(5): p. 1538-1539.

[28] Fleury, E.d.F.C., et al., New elastographic classification of breast lesions during and after compression. Diagnostic and Interventional Radiology, 2009. 15(2): p. 96. 